\title{
Effectiveness and Safety of Apixaban, Low-Molecular-Weight Heparin, and Warfarin among Venous Thromboembolism Patients with Active Cancer: A U.S. Claims Data Analysis
}

\author{
Alexander Cohen ${ }^{1}$ Allison Keshishian ${ }^{2}$ Theodore Lee ${ }^{3}$ Gail Wygant ${ }^{4}$ Lisa Rosenblatt ${ }^{4}$ \\ Patrick Hlavacek ${ }^{3}$ Jack Mardekian $^{3}$ Daniel Wiederkehr ${ }^{3}$ Janvi Sah ${ }^{2}$ Xuemei Luo ${ }^{5}$
}

\footnotetext{
${ }^{1}$ Department of Hematological Medicine, Guy's \& St Thomas' NHS Foundation Trust, King's College London, Westminster Bridge Road, London, United Kingdom

2 SIMR, LLC, Ann Arbor, Michigan, United States

3 Pfizer Inc., New York, New York, United States

${ }^{4}$ Bristol-Myers Squibb Company, Lawrenceville, New Jersey, United States

5 Pfizer Inc., Groton, Connecticut, United States
}

\author{
Address for correspondence Alexander Cohen, MD, Department of \\ Hematological Medicine, Guy's and St Thomas' Hospitals, King's \\ College London, London WC2R 2LS, United Kingdom \\ (e-mail: alexander.cohen@kcl.ac.uk).
}

Thromb Haemost 2021;121:383-395.

received

May 4, 2020

accepted after revision

September 2, 2020

published online

November 10, 2020
Background This study primarily evaluates the risk of recurrent venous thromboembolism (VTE) and major bleeding (MB) among patients with VTE and active cancer prescribed apixaban, low-molecular-weight heparin (LMWH), or warfarin, with claims data.

Methods Four U.S. commercial insurance claims databases were used to identify patients with VTE and active cancer who initiated apixaban, LMWH, or warfarin within 30 days following the first VTE event. Stabilized inverse-probability treatment weighting (IPTW) was used to balance treatment cohorts. Cox proportional hazard models were used to evaluate risk of recurrent VTE and MB.

Results All eligibility criteria were fulfilled by 3,393 apixaban, 6,108 LMWH, and 4,585 warfarin patients. After IPTW, all patient characteristics were balanced. When the follow-up was censored at 6 months, apixaban patients had a lower risk of recurrent VTE (hazard ratio [HR]: 0.61; 95\% confidence interval [Cl]: 0.47-0.81) and MB (HR: 0.63; 95\% Cl: 0.47-0.86) versus LMWH. Apixaban patients had a lower risk of recurrent VTE (HR: $0.68 ; 95 \% \mathrm{Cl}$ : $0.52-0.90)$ and similar risk of MB (HR: $0.73 ; 95 \% \mathrm{Cl}: 0.53-1.00)$ versus warfarin. Warfarin patients had a similar risk of recurrent VTE (HR: $0.91 ; 95 \% \mathrm{Cl}: 0.72-1.15)$ and MB (HR: 0.87; 95\% Cl: 0.68-1.12) versus LMWH. The trends were similar for the entire follow-up; however, apixaban patients had a lower risk of MB versus warfarin patients.

Conclusion Patients with VTE and active cancer who initiated apixaban had a lower risk of recurrent VTE and MB compared with LMWH patients. Apixaban patients also had a lower risk of recurrent VTE compared with warfarin patients.
DOI https://doi.org/ $10.1055 / \mathrm{s}-0040-1718728$. ISSN 0340-6245. (c) 2020. The Author(s).

This is an open access article published by Thieme under the terms of the Creative Commons Attribution-NonDerivative-NonCommercial-License, permitting copying and reproduction so long as the original work is given appropriate credit. Contents may not be used for commercial purposes, or adapted, remixed, transformed or built upon. (https://creativecommons.org/ licenses/by-nc-nd/4.0/)

Georg Thieme Verlag KG, Rüdigerstraße 14, 70469 Stuttgart, Germany 


\section{Introduction}

Venous thromboembolism (VTE), including deep vein thrombosis (DVT) or pulmonary embolism (PE), is a leading cause of death in cancer patients receiving chemotherapy. ${ }^{1}$ Cancer is an independent risk factor for VTE, accounting for $18 \%$ of the total VTE cases, and it is the strongest predictor for all-cause and PE-related mortality in VTE. ${ }^{2,3}$ The risk of VTE depends on patient characteristics such as age, cancer stage, type of malignancy, and cancer treatment. ${ }^{4,5}$ Cancer treatments such as cancer surgery and radiation therapy were found to be associated with VTE. 6,7 Breast, lung, colon, and prostate cancers contribute the most to the burden of active cancer-associated VTE. ${ }^{8}$ Cancer-associated VTE carries a significantly greater risk of recurrent VTE and major bleeding (MB) compared with VTE in noncancer patients. ${ }^{9,10}$ Given the risk of recurrent VTE following the initial 3 months of anticoagulant therapy, patients with VTE and active cancer usually require $\geq 6$ months of anticoagulation treatment and should be considered for extended treatment until the cancer is cured or quiescent in those who do not have a high bleeding risk. ${ }^{11,12}$

Treating VTE patients with cancer is challenging due to an increased risk of bleeding associated with anticoagulant use and potential cancer treatment complications such as drug-drug interactions. ${ }^{10,13}$ The recent National Comprehensive Cancer Network (NCCN) Clinical Practice Guidelines in Oncology (NCCN Guidelines) and International Society on Thrombosis and Haemostasis (ISTH) Scientific and Standardization Committee recommend the use of $\mathrm{LMWH}$, edoxaban, and rivaroxaban for the treatment of cancer-associated VTE. ${ }^{14,15}$ The American Society of Clinical Oncology suggests the use of LMWH for $\geq 6$ months in VTE cancer patients and recommends the use of indefinite anticoagulation therapy among active cancer patients, such as those with metastatic disease and those receiving chemotherapy. ${ }^{16}$ The European Society of Cardiology recommends similar considerations for the treatment of PE in cancer patients. ${ }^{12}$ Despite the recommendation to use LMWH and specific direct-acting oral anticoagulants (DOACs) in VTE patients with cancer, real-world data show that warfarin and a variety of DOACs are being used in practice to treat these patients. ${ }^{17}$ Clinical trials and real-world studies have assessed DOACs including rivaroxaban and edoxaban versus LMWH for the treatment of cancer-associated VTE. ${ }^{18-20}$ However, there is limited evidence for apixaban in VTE cancer patients. CARAVAGGIO, a recently completed multinational prospective, randomized, open-label, blinded end point (PROBE), noninferiority study found that apixaban was noninferior to dalteparin for the treatment of cancer-associated VTE without an increased risk of $\mathrm{MB} .{ }^{21}$ The ADAM trial of 300 randomized patients reported that apixaban was associated with low rates of bleeding and VTE recurrence compared with dalteparin in treating cancer-associated VTE. ${ }^{22}$ Despite these clinical trials, there is a lack of realworld evidence comparing the effectiveness and safety of LMWH with vitamin K antagonists (VKAs) and apixaban among patients with VTE and active cancer. Using four U.S. claims databases, this study compared the risk of recurrent VTE, MB, and clinically relevant nonmajor (CRNM) bleeding among patients with VTE and active cancer who newly initiated apixaban, LMWH, or warfarin in routine clinical practice.

\section{Methods}

\section{Data Source and Patient Selection}

Data on this study were pooled from four U.S. commercial claims databases: the IBM MarketScan Commercial Claims and Encounter and Medicare Supplemental and Coordination of Benefits Database (MarketScan), IQVIA PharMetrics Plus (PharMetrics), Optum Clinformatics Data Mart (Optum), and the Humana Research Database (Humana). These databases contain medical and pharmacy claims for commercial and Medicare populations in the United States. The medical claims are coded using International Classification of Disease, Ninth Revision, Clinical Modification (ICD-9-CM), ICD-10-CM (implemented October 01, 2015), Current Procedural Terminology, or Health Care Common Procedure Coding System codes, and the National Drug Code coding system was used for the pharmacy claims. This study was registered at the EU Post Authorization Study register http:// www.encepp.eu/encepp/studiesDatabase.jsp (EU PAS registration number: EUPAS25308).

Patient selection criteria are described in - Fig. 1. Patients with $\geq 1$ medical claim for VTE in the inpatient or outpatient setting ("index VTE event") from September 01, 2014 to the end of the study period (identification period) and a diagnosis for active cancer (defined as having $\geq 2$ medical claims for cancer diagnosis [exclude nonmelanoma skin cancer] or 1 claim for cancer diagnosis plus $\geq 1$ claim for cancer treatment [e.g., chemotherapy, radiation, immunotherapy, cancer-related surgery] within the time period 6 months before until 30 days after the index VTE event) were identified. The end of study was different for each database based on the last available at the time of analysis (MarketScan: March 01, 2014-June 30, 2017; Optum \& Humana: March 01, 2014December 31, 2017; PharMetrics: March 01, 2014-March 31, 2018). Adult patients (aged $\geq 18$ years) with $\geq 1$ pharmacy claim for apixaban, LMWH, or warfarin within 30 days following the index VTE event were classified into the following cohorts:

- LMWH Cohort: If VTE patients had LMWH for $\geq 14$ days after the index VTE event and did not have another anticoagulant during the period between the index VTE event and 14 days after LMWH initiation, then the first LMWH prescription date was designated as the index date.

- Warfarin Cohort: For VTE patients with a warfarin claim within 30 days after the index VTE event and without a claim for any other anticoagulant (except for LMWH as a bridging therapy) between the index VTE event and the warfarin initiation date, the first warfarin prescription date was designated as the index date. The LMWH bridging therapy was defined as having a claim for LMWH within 14 days before or after warfarin initiation and LMWH duration of $\leq 14$ days. 
Had claims indicating a VTE diagnosis ('index VTE event') in the primary or secondary position in an inpatient or outpatient setting during the identification period (01SEPT2014 to end of study period ${ }^{\mathrm{a}}$ );

$$
N=1,124,562
$$

Had $\geq 2$ medical claims for cancer diagnosis or 1 claim for cancer diagnosis and $\geq 1$ claim for cancer treatment 6 months before or 30 days after index VTE event;

$$
N=173,853
$$

Had claims for apixaban, LMWH, or warfarin during the 30-day period after the index VTE event; the date of first prescription that met the criteria was designated as the index date;

$$
N=57,436
$$

Were aged $\geq 18$ years and had continuous health plan enrollment with medical and pharmacy benefits for 6 months prior to the index VTE event until the index date;

$$
N=32,889
$$



Fig. 1 Patient selection criteria. LMWH, low-molecular-weight heparin; OAC, oral anticoagulant; PAC, parenteral anticoagulant; VTE, venous thromboembolism. 'adentification period: (MarketScan: September 01, 2014-June 30, 2017; Optum \& Humana: September 01, 2014December 31, 2017; PharMetrics: September 01, 2014-March 31, 2018).

- Apixaban Cohort: For VTE patients who initiated apixaban within 30 days after the index VTE event and did not have a claim for any other anticoagulant between the index VTE event and the initiation of apixaban, the first apixaban prescription date was designated as the index date.

Patients were also required to have continuous health plan enrollment for 6 months prior to the index VTE event as well as during the time between the index VTE event and the index date. The baseline period was defined as 6 months prior to the index date (inclusive of the index date). Patients were excluded from the study if they had any evidence of atrial fibrillation/flutter or a mechanical heart valve within 6 months prior to the index date, diagnosis of VTE, oral anticoagulant $(\mathrm{OAC})$ /parenteral anticoagulant (PAC) use (unless the therapy was administered prophylactically) 
during the 6 months preceding the index VTE event, did not have outpatient OAC/PAC during the period between the index VTE event and index date, did not have another OAC/PAC on the index date, or evidence of inferior vena cava filter/pregnancy anytime during the study period. Among patients who had LMWH as bridging therapy before warfarin initiation, patients who had a recurrent VTE or MB event (defined in the outcomes below) between LMWH and warfarin initiation were excluded.

Patients were followed from the day after the index date through the earliest of the following: health plan disenrollment, death, index therapy discontinuation, switch to a nonindex OAC/PAC, or study end. Analyses were first conducted by censoring patients at 6 months of follow-up. Additional analyses were conducted using the entire follow-up period. Discontinuation was defined as no evidence of the index apixaban, LMWH, or warfarin prescription for 30 days from the last day of supply of the last filled prescription. ${ }^{23}$ The date of discontinuation was defined as 30 days after the last day of the last filled prescription's days' supply. Switch was defined as a prescription for an OAC other than the index OAC prescription within 30 days before or after the last days' supply of the index OAC prescription. $^{24}$ For the warfarin cohort, a LMWH claim was allowed within 14 days after the index date if the days of supply for LMWH were $\leq 14$ days, as LMWH was considered to be bridging therapy for warfarin in this case.

\section{Outcome Measures}

Treatment patterns that were evaluated included persistence, discontinuation, and switch. Persistence (in days) was defined as the number of days the patient remained on the index drug with a gap of $\leq 30$ days between the runout date of the previous prescription and the following prescription. Patients who were persistent included those who were neither discontinued nor switched the index drug. Percentage of patients who were persistent, discontinued, or switched the index drug was evaluated. Risk of nonpersistent (discontinued or switched) was also examined.

Outcome measures were recurrent VTE, MB, and CRNM bleeding. Recurrent VTE and MB events were identified based on inpatient claims with VTE or MB as the first-listed diagnosis. The ICD diagnosis codes (not blood tests) used to identify recurrent VTE have been validated in previous studies with a positive predictive value ranging from 26 to $93 \% .^{25,26}$ If the admissions for recurrent VTE occurred within 7 days of the index VTE event, irrespective of care setting, the events were not considered as recurrent VTE events due to the proximity to the index VTE event. MB included gastrointestinal (GI) bleeding, intracranial hemorrhage (ICH), and bleeding at other sites (genitourinary bleeding, respiratory tract bleeding, ocular bleeding, joint bleeding/hemarthrosis, transfusion of blood and blood components, other bleeding, or no bleeding site specified). A CRNM bleeding event was defined as a noncritical site bleeding that did not qualify as MB but required either hospitalization with the bleeding as a secondary diagnosis or an outpatient visit (including emergency department visits). Specifically, it was defined as (1) an inpatient admission with a secondary diagnosis for "noncritical site" bleeding such as GI bleeding or other selected noncritical types/sites of bleeding (excluded if MB occurred during the same hospitalization), or (2) an outpatient encounter with a diagnosis code for GI bleeding and other selected noncritical types/sites of bleeding. CRNM bleeding events that followed a MB event were not included in the analysis of CRNM bleeding. All the clinical outcomes were measured independently; patients were censored upon recurrent VTE, MB, or CRNM bleeding events for the respective analysis. Outcomes and treatment patterns were assessed first by censoring patients at 6 months of follow-up and then using the entire follow-up period.

Patient demographics, clinical characteristics (baseline comorbidities and medications), VTE-related variables, cancer site and type, VTE risk scale, and cancer-related treatment were measured during the baseline period. A modified Khorana VTE risk scale (based on ICD codes and not blood tests) was used to evaluate the proportion of patients with very high risk (brain, stomach, or pancreas cancer), high risk (lung, lymphoma, gynecologic, bladder, testicular, or renal cell carcinoma cancer), or other cancers. ${ }^{27}$

\section{Statistical Methods}

Inverse-probability treatment weighting (IPTW) was used to balance patient characteristics between apixaban, LMWH, and warfarin cohorts. ${ }^{28}$ Propensity scores were used to obtain estimates of the average treatment effect using a multinomial logistic model with the three treatment cohorts and LMWH patients as the reference (i.e., control cohort). Covariates including demographics, type of VTE diagnosis, VTE etiology (provoked vs. unprovoked), modified Charlson comorbidity index (CCI) score (did not include cancer), ${ }^{29}$ comorbidities, medication use, cancer metastases, modified Khorana VTE risk scale, and cancer-related treatment were used to define the probability of a patient receiving a certain treatment. After the propensity score calculation, each patient was weighted by the inverse of the probability of their treatment option (weight $=1$ /propensity score). The weights were stabilized by multiplying the original weights with a constant, which is equal to the expected value of being in the treatment or comparison cohorts, respectively. ${ }^{30-32}$ The baseline characteristics were well balanced in each of the four databases after IPTW, and patients were pooled from the four databases for further analysis.

After IPTW, incidence rates of recurrent VTE, MB, and CRNM bleeding were calculated as the number of events per 100 person-years among the weighted population. The risk of recurrent VTE, MB, and CRNM bleeding in each weighted cohort was evaluated using a Cox proportional hazard model and Kaplan-Meier (KM) survival curves. The cohort was included as an independent variable, and no covariates were included in the models as they were balanced. All the analyses were conducted first by censoring the follow-up at 6 months and then using the entire follow-up.

\section{Results}

After applying the selection criteria, a total of 14,086 patients with VTE and active cancer were identified, including 3,393 
(24.1\%) apixaban patients, 6,108 (43.4\%) LMWH patients, and $4,585(32.5 \%)$ warfarin patients in the pooled analysis (-Fig. 1). Before IPTW, warfarin patients were older and had the highest baseline CCI followed by apixaban and LMWH (- Supplementary Table S1 [available in the online version]). A total of 9.5\%, 20.6\%, and 9.6\% of apixaban, $\mathrm{LMWH}$, and warfarin patients, respectively, were categorized as having very high risk cancer types. Most LMWH patients (88.7\%) received cancer-related treatment, as did warfarin (63.6\%) and apixaban (68.4\%) initiators (-Supplementary Table s1, available in the online version). After applying IPTW, all patient characteristics were balanced (-Supplementary Table $\mathbf{5 2}$ [available in the online version]). In the weighted population, approximately $34 \%$ of patients had history of baseline bleed, $51 \%$ had metastatic cancer, and $63 \%$ received chemotherapy during the baseline period (-Table 1). Further, 15\% had very high risk cancer and $40 \%$ had high-risk cancer.

\section{Treatment Patterns}

Among the IPTW-weighted cohorts, apixaban had the highest persistence when the follow-up was censored at
6 months, followed by warfarin and LMWH (-Fig. 2). Apixaban (hazard ratio [HR]: 0.52; 95\% confidence interval [CI]: 0.48-0.56) and warfarin (HR: 0.60; 95\% CI: 0.56-0.64) patients had a lower risk of nonpersistence compared with LMWH patients when the follow-up was censored at 6 months (-Supplementary Table s3 [available in the online version]). Additionally, apixaban patients also had a lower risk of nonpersistence compared with warfarin patients (HR: 0.87; 95\% CI: 0.80-0.94). Similar trends were seen when the entire follow-up was used (-Supplementary Table $\mathbf{5 3}$ [available in the online version]).

\section{Clinical Outcomes Censoring Follow-Up at 6 Months}

In the IPTW population, the mean follow-up was 105 days (3.5 months), 88 days (2.9 months), and 113 days (3.8 months) for apixaban, LMWH, and warfarin, respectively, when patients censored at 6 months of follow-up. The adjusted incidence rate of recurrent VTE was 15.8 (apixaban), 28.8 (LMWH), and 22.2 (warfarin) per 100 personyears. The adjusted incidence rate of MB-including GI, ICH, and other bleeding-was 11.8 (apixaban), 20.1 (LMWH),

Table 1 IPTW-weighted patient characteristics among VTE cancer patients prescribed apixaban, LMWH, or warfarin

\begin{tabular}{|c|c|c|c|c|c|c|c|c|}
\hline & \multicolumn{2}{|c|}{$\begin{array}{l}\text { LMWH cohort } \\
\text { (reference) }\end{array}$} & \multicolumn{3}{|c|}{ Warfarin cohort } & \multicolumn{3}{|c|}{ Apixaban cohort } \\
\hline & $N /$ mean & $\% / S D$ & $N /$ mean & $\% / S D$ & STD $^{a}$ & $N /$ mean & $\% / S D$ & STD $^{a}$ \\
\hline Sample size & 6,108 & & 4,585 & & & 3,393 & & \\
\hline $\mathrm{Age}^{\mathrm{b}}$ & 63.7 & 13.2 & 64.2 & 12.9 & 3.5 & 64.6 & 12.6 & 7.1 \\
\hline $18-54$ & 1,247 & $20.4 \%$ & 967 & $21.1 \%$ & 1.7 & 660 & $19.4 \%$ & 2.4 \\
\hline $55-64$ & 1,970 & $32.3 \%$ & 1,475 & $32.2 \%$ & 0.2 & 1,105 & $32.6 \%$ & 0.7 \\
\hline $65-74$ & 1,531 & $25.1 \%$ & 1,139 & $24.9 \%$ & 0.5 & 875 & $25.8 \%$ & 1.7 \\
\hline $75-79$ & 636 & $10.4 \%$ & 462 & $10.1 \%$ & 1.1 & 353 & $10.4 \%$ & 0.1 \\
\hline$\geq 80$ & 723 & $11.8 \%$ & 541 & $11.8 \%$ & 0.1 & 400 & $11.8 \%$ & 0.1 \\
\hline \multicolumn{9}{|l|}{ Gender $^{c}$} \\
\hline Male & 2,869 & $47.0 \%$ & 2,171 & $47.3 \%$ & 0.7 & 1,621 & $47.8 \%$ & 1.6 \\
\hline Female & 3,237 & $53.0 \%$ & 2,412 & $52.6 \%$ & 0.8 & 1,772 & $52.2 \%$ & 1.6 \\
\hline \multicolumn{9}{|l|}{ Setting of index VTE event } \\
\hline Inpatient & 3,017 & $49.4 \%$ & 2,313 & $50.4 \%$ & 2.1 & 1,679 & $49.5 \%$ & 0.2 \\
\hline Outpatient & 3,091 & $50.6 \%$ & 2,272 & $49.6 \%$ & 2.1 & 1,714 & $50.5 \%$ & 0.2 \\
\hline \multicolumn{9}{|l|}{ VTE diagnosis } \\
\hline DVT only & 3,605 & $59.0 \%$ & 2,643 & $57.6 \%$ & 2.8 & 1,938 & $57.1 \%$ & 3.9 \\
\hline PE with or without DVT & 2,503 & $41.0 \%$ & 1,942 & $42.4 \%$ & 2.8 & 1,455 & $42.9 \%$ & 3.9 \\
\hline \multicolumn{9}{|l|}{ Baseline comorbidity } \\
\hline Deyo-Charlson comorbidity index ${ }^{\mathrm{d}}$ & 2.0 & 2.1 & 1.9 & 2.0 & 1.9 & 2.0 & 2.0 & 0.5 \\
\hline Central venous catheter & 1,790 & $29.3 \%$ & 1,314 & $28.7 \%$ & 1.4 & 985 & $29.0 \%$ & 0.6 \\
\hline Cerebrovascular disease & 712 & $11.7 \%$ & 528 & $11.5 \%$ & 0.5 & 391 & $11.5 \%$ & 0.4 \\
\hline Coagulation defects & 894 & $14.6 \%$ & 657 & $14.3 \%$ & 0.9 & 503 & $14.8 \%$ & 0.6 \\
\hline Ischemic heart/coronary artery disease & 1,182 & $19.3 \%$ & 888 & $19.4 \%$ & 0.0 & 667 & $19.7 \%$ & 0.8 \\
\hline Dyspepsia or stomach discomfort & 2,205 & $36.1 \%$ & 1,629 & $35.5 \%$ & 1.2 & 1,222 & $36.0 \%$ & 0.2 \\
\hline
\end{tabular}


Table 1 (Continued)

\begin{tabular}{|c|c|c|c|c|c|c|c|c|}
\hline & \multicolumn{2}{|c|}{$\begin{array}{l}\text { LMWH cohort } \\
\text { (reference) }\end{array}$} & \multicolumn{3}{|c|}{ Warfarin cohort } & \multicolumn{3}{|c|}{ Apixaban cohort } \\
\hline & $N /$ mean & $\% / S D$ & $N /$ mean & $\% / S D$ & STD $^{a}$ & $N /$ mean & $\% / S D$ & STD $^{a}$ \\
\hline Hemiplegia or paraplegia & 171 & $2.8 \%$ & 126 & $2.8 \%$ & 0.3 & 93 & $2.7 \%$ & 0.4 \\
\hline Hyperlipidemia & 2,424 & $39.7 \%$ & 1,864 & $40.6 \%$ & 2.0 & 1,391 & $41.0 \%$ & 2.7 \\
\hline Obesity & 1,118 & $18.3 \%$ & 843 & $18.4 \%$ & 0.2 & 615 & $18.1 \%$ & 0.5 \\
\hline Pneumonia & 1,040 & $17.0 \%$ & 791 & $17.2 \%$ & 0.6 & 586 & $17.3 \%$ & 0.7 \\
\hline Sleep apnea & 593 & $9.7 \%$ & 460 & $10.0 \%$ & 1.1 & 365 & $10.8 \%$ & 3.5 \\
\hline Thrombophilia & 328 & $5.4 \%$ & 268 & $5.9 \%$ & 2.1 & 210 & $6.2 \%$ & 3.5 \\
\hline Congestive heart failure & 676 & $11.1 \%$ & 511 & $11.1 \%$ & 0.3 & 366 & $10.8 \%$ & 0.9 \\
\hline Diabetes & 1,637 & $26.8 \%$ & 1,219 & $26.6 \%$ & 0.5 & 907 & $26.7 \%$ & 0.2 \\
\hline Hypertension & 3,834 & $62.8 \%$ & 2,860 & $62.4 \%$ & 0.8 & 2,170 & $64.0 \%$ & 2.5 \\
\hline Liver disease & 1,334 & $21.8 \%$ & 968 & $21.1 \%$ & 1.8 & 770 & $22.7 \%$ & 2.0 \\
\hline Chronic obstructive pulmonary disease & 1,253 & $20.5 \%$ & 913 & $19.9 \%$ & 1.5 & 667 & $19.7 \%$ & 2.2 \\
\hline Baseline any bleed & 2,088 & $34.2 \%$ & 1,575 & $34.3 \%$ & 0.3 & 1,132 & $33.4 \%$ & 1.8 \\
\hline Recent history of falls & 259 & $4.2 \%$ & 176 & $3.8 \%$ & 2.1 & 147 & $4.3 \%$ & 0.5 \\
\hline Fracture/trauma involving lower extremities & 530 & $8.7 \%$ & 364 & $7.9 \%$ & 2.7 & 288 & $8.5 \%$ & 0.7 \\
\hline Selected surgeries & 2,966 & $48.6 \%$ & 2,105 & $45.9 \%$ & 5.3 & 1,586 & $46.7 \%$ & 3.7 \\
\hline Cancer metastasis $^{\mathrm{e}}$ & 3,172 & $51.9 \%$ & 2,344 & $51.1 \%$ & 1.6 & 1,727 & $50.9 \%$ & 2.1 \\
\hline \multicolumn{9}{|l|}{ Cancer type $e^{e, f}$} \\
\hline Hematological & 992 & $16.2 \%$ & 749 & $16.3 \%$ & 0.3 & 543 & $16.0 \%$ & 0.7 \\
\hline Nonhematological & 5,116 & $83.8 \%$ & 3,830 & $83.5 \%$ & 0.6 & 2,846 & $83.9 \%$ & 0.3 \\
\hline \multicolumn{9}{|l|}{ VTE risk scale } \\
\hline Very high risk ${ }^{g}$ & 921 & $15.1 \%$ & 708 & $15.4 \%$ & 1.0 & 518 & $15.3 \%$ & 0.5 \\
\hline High risk $^{\mathrm{h}}$ & 2,502 & $41.0 \%$ & 1,849 & $40.3 \%$ & 1.3 & 1,343 & $39.6 \%$ & 2.8 \\
\hline Other cancers & 2,685 & $44.0 \%$ & 2,028 & $44.2 \%$ & 0.6 & 1,532 & $45.2 \%$ & 2.4 \\
\hline \multicolumn{9}{|l|}{ Cancer-related treatment $^{\mathrm{e}}$} \\
\hline $\begin{array}{l}\text { Number of patients that had } \\
\text { cancer-related treatment } \\
\text { during the baseline period until } \\
30 \text { days after the index date }\end{array}$ & 4,722 & $77.3 \%$ & 3,494 & $76.2 \%$ & 2.6 & 2,560 & $75.4 \%$ & 4.4 \\
\hline Chemotherapy & 3,895 & $63.8 \%$ & 2,889 & $63.0 \%$ & 1.6 & 2,139 & $63.0 \%$ & 1.5 \\
\hline Hormone therapy & 364 & $6.0 \%$ & 270 & $5.9 \%$ & 0.3 & 201 & $5.9 \%$ & 0.2 \\
\hline Immunotherapy & 125 & $2.0 \%$ & 94 & $2.0 \%$ & 0.0 & 64 & $1.9 \%$ & 1.3 \\
\hline Radiation & 2197 & $36.0 \%$ & 1638 & $35.7 \%$ & 0.5 & 1,180 & $34.8 \%$ & 2.5 \\
\hline Cancer-related surgery & 775 & $12.7 \%$ & 611 & $13.3 \%$ & 1.9 & 430 & $12.7 \%$ & 0.1 \\
\hline
\end{tabular}

Abbreviations: DVT, deep vein thrombosis; LMWH, low-molecular-weight heparin; PE, pulmonary embolism; SD, standard deviation; STD, standardized difference; VTE, venous thromboembolism.

${ }^{\text {a STD }}=100 \times \mid$ actual STD $\mid$. STD $>10.00$ is considered significant.

${ }^{\mathrm{b}}$ After applying weights, the values for age category were not whole numbers; therefore, due to rounding the sum of patients does not equal $100 \%$. 'Some patients in Optum and PharMetrics data have missing information on gender. Hence, the sum of male and female is not equal to $100 \%$.

${ }^{\mathrm{d}} \mathrm{A}$ modified comorbidity index was used which included myocardial infarction, congestive heart failure, peripheral vascular disease, cerebrovascular disease, dementia, rheumatologic disease, peptic ulcer disease, mild liver disease, diabetes, diabetes w/ complications, hemiplegia or paraplegia, renal disease, moderate or severe liver disease, and acquired immune deficiency syndrome.

${ }^{\text {e}}$ Cancer-related variables will be measured 6 months prior to the index date until 30 days after the index date.

${ }^{f}$ The sum of hematological and nonhematological cancer was not equal to $100 \%$ since a very small number of patients had a cancer diagnosis at month 6 before the index VTE event (month 7 before the index date) and not captured in the baseline period.

${ }^{9}$ Very high risk (brain, stomach, and pancreas).

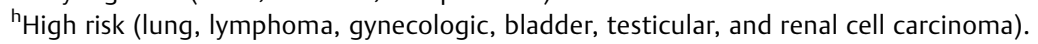


Effectiveness of Apixaban, LMWH, and Warfarin among Patients with VTE and Active Cancer Cohen et al. 389

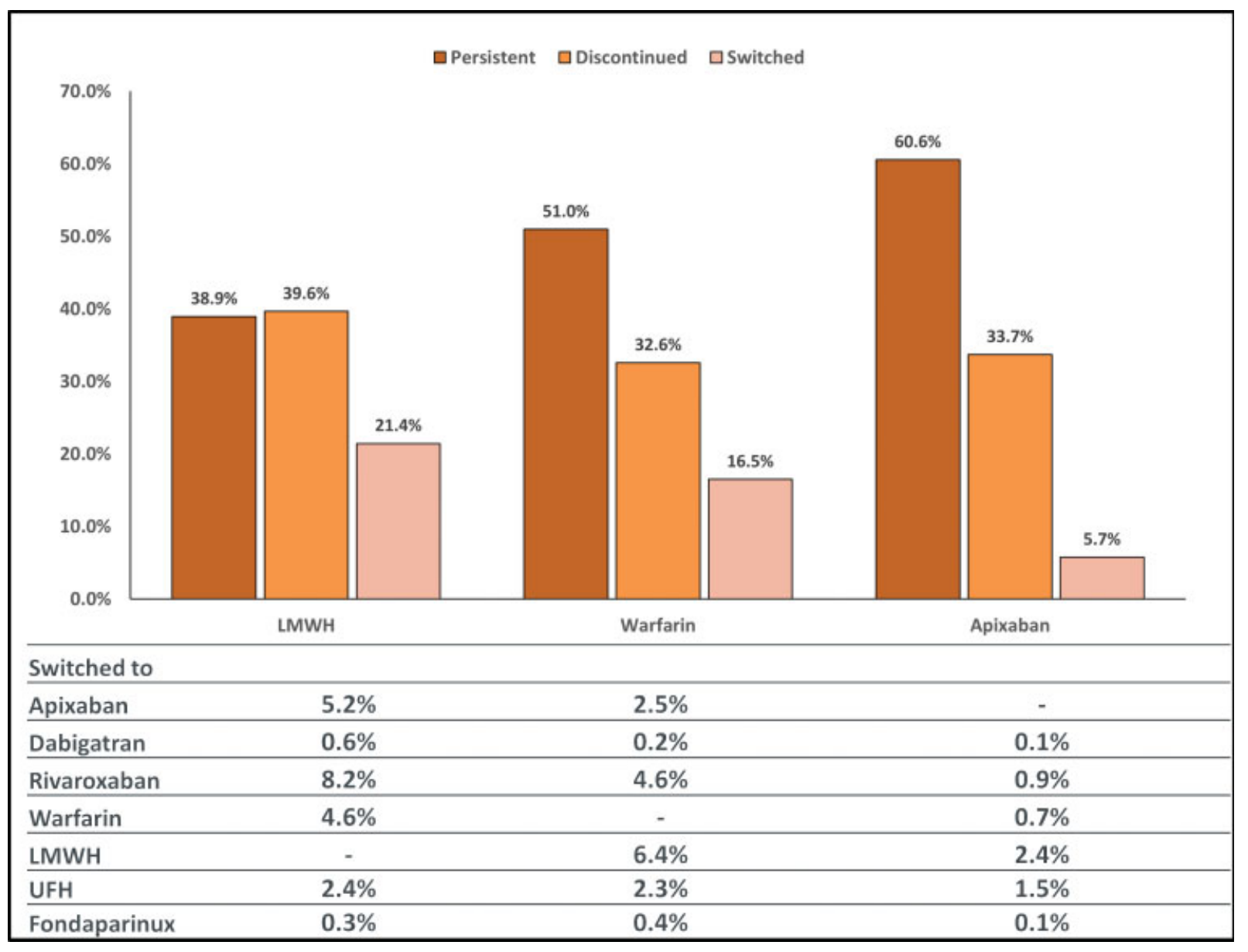

Fig. 2 Treatment patterns among VTE cancer patients initiated apixaban, LMWH, and warfarin in the IPTW-weighted population censoring follow-up at 6 months. IPTW, inverse probability of treatment weighing; LMWH, low-molecular-weight heparin; UFH, unfractionated heparin; VTE, venous thromboembolism.

and 15.7 (warfarin) per 100 person-years when the followup was censored at 6 months. The KM curves for cumulative incidence rates for recurrent VTE, MB, and CRNM bleeding censoring the follow-up at 6 months and the entire follow-up in the weighted population are shown in -Fig. 3.

Compared with LMWH, apixaban had a lower risk of recurrent VTE (HR: 0.61; 95\% CI: 0.47-0.81), MB (HR: 0.63; 95\% CI: 0.47-0.86), and CRNM bleeding (HR: 0.81; 95\% CI: 0.70-0.94; - Fig. 4). Warfarin patients had a similar risk of recurrent VTE (HR: 0.91; 95\% CI: 0.72-1.15), MB (HR: 0.87; 95\% CI: 0.68-1.12), and CRNM bleeding (HR: 0.90; 95\% CI: 0.79-1.04) compared with LMWH patients. Apixaban patients had a lower risk of recurrent VTE (HR: 0.68; 95\% $\mathrm{CI}: 0.52-0.90)$ but a similar risk of $\mathrm{MB}$ (HR: $0.73 ; 95 \% \mathrm{CI}$ : $0.53-1.00$ ) and CRNM bleeding (HR: 0.89; 95\% CI: 0.77-1.04) compared with warfarin patients (-Fig. 4).

\section{Clinical Outcomes during the Entire Follow-up}

When the entire available follow-up was evaluated in the IPTW-weighted population, the mean follow-up was 137 days (4.6 months), 105 days (3.5 months), and 166 days (5.5 months) for the apixaban, LMWH, and warfarin cohorts, respectively. The maximum length of follow-up was approximately 3 years for apixaban and LMWH and 3.3 years for warfarin. - Fig. 3 shows the KM curves for cumulative incidence rates for recurrent VTE, $\mathrm{MB}$, and CRNM bleeding over the entire follow-up period. Findings when using the entire follow-up period were generally consistent compared with the outcomes when follow-up was censored at 6 months (-Fig. 5). One difference is that apixaban patients had a significantly lower risk of $\mathrm{MB}$ (HR: 0.72; 95\% CI: 0.55-0.95) compared with warfarin patients during the entire follow-up (-Fig. 5).

\section{Discussion}

This study, pooling four large US commercial claims databases, demonstrated that in treatment of patients with cancer-associated VTE apixaban was associated with significantly lower risks of recurrent VTE, MB, and CRNM bleeding compared with LMWH. Additionally, apixaban was associated with a lower risk of recurrent VTE compared with warfarin initiators. Warfarin was associated with similar risks of recurrent VTE, MB, and CRNM bleeding compared with LMWH patients. Findings were consistent when the follow-up was censored at 6 months and 

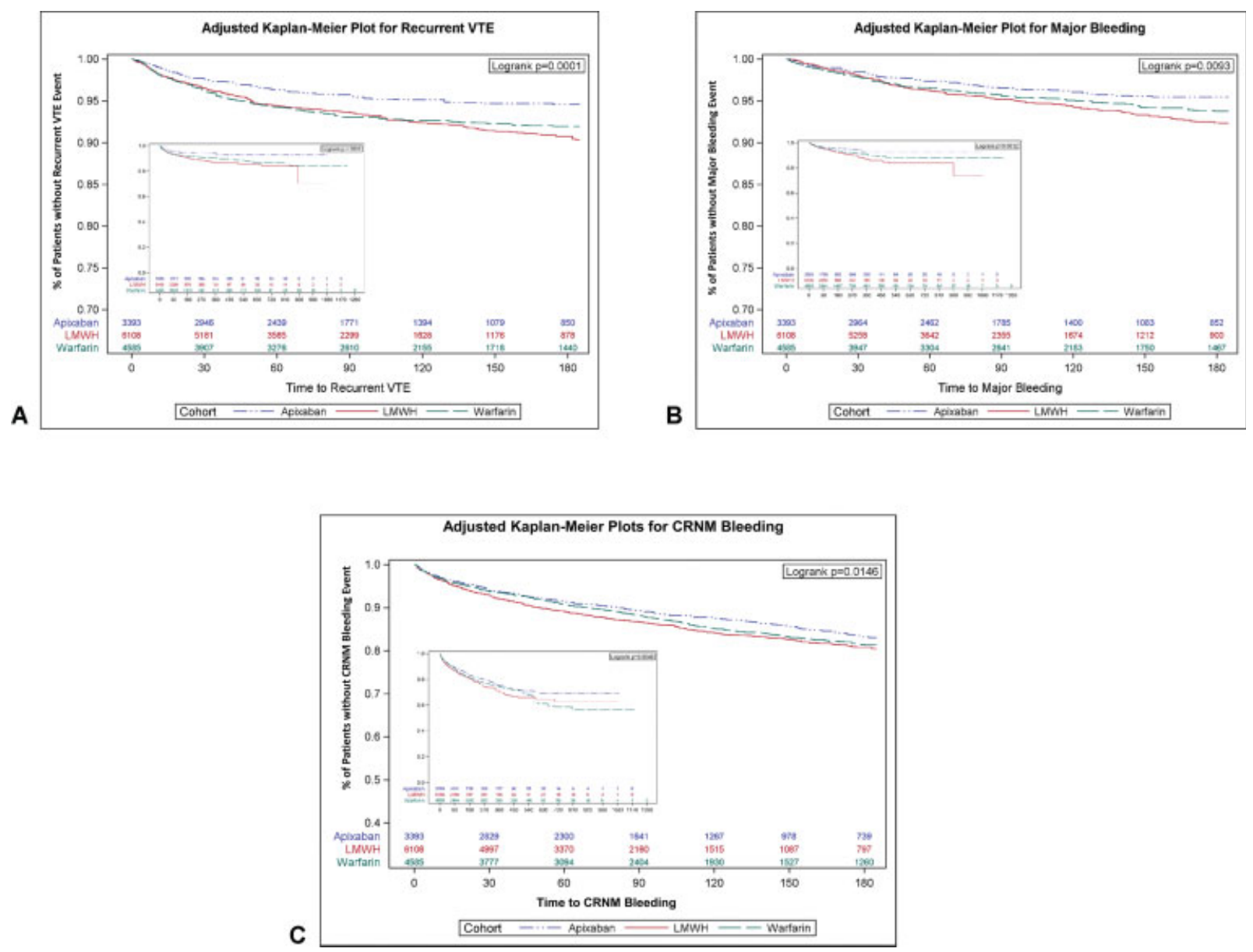

Fig. 3 (A) Cumulative incidence of recurrent VTE among VTE cancer patients prescribed apixaban, LMWH, or warfarin in the IPTW-weighted population censoring follow-up at 6 months or during the entire follow-up (insert). (B) Cumulative incidence of major bleeding among VTE cancer patients prescribed apixaban, LMWH, or warfarin in the IPTW-weighted population censoring follow-up at 6 months or during the entire followup (insert). (C) Cumulative incidence of CRNM bleeding among VTE cancer patients prescribed apixaban, LMWH, or warfarin in the IPTWweighted population censoring follow-up at 6 months or during the entire follow-up (insert). CRNM, clinically relevant nonmajor (bleeding); IPTW, inverse probability weighting; LMWH, low-molecular-weight heparin; VTE, venous thromboembolism.

when the entire follow-up was used to evaluate the outcomes.

The clinical guidelines, until very recently, had recommended primarily the use of LMWH for the first 6 months for the treatment of VTE in cancer patients. Despite the recommendation, LMWH remains underutilized. In a review of published surveys, registries, and observational studies, only $50 \%$ of patients were treated with LMWH for cancer-associated VTE. ${ }^{33}$ Additionally, a real-world study among commercially insured patients showed that $50 \%$ of the cancer patients who developed VTE used warfarin, $40 \%$ used LMWH, and approximately $10 \%$ used DOACs or fondaparinux. ${ }^{17}$ The study also reported that during the 6 months of observation, $44 \%$ of LMWH patients and $28 \%$ of warfarin patients switched to other anticoagulants. ${ }^{17}$ In our study, a higher proportion of LMWH (21.4\%) patients switched their index treatment compared with apixaban (5.7\%) and warfarin (16.5\%) patients. Nonadherence to clinical guidelines could be due to the inconvenience associated with the use of LMWH, risk of bleeding, reluc- tance to impose daily injections on fragile patients, and personal preference. ${ }^{31,34}$ DOACs, on the other hand, offer quick onset of action, higher bioavailability, and shorter half-lives compared with warfarin; additionally, rivaroxaban and apixaban do not require concomitant LMWH therapy. ${ }^{35,36}$

The choice of anticoagulation among VTE cancer patients is based on a balance between the risk of bleeding and of VTE recurrence. The recommendation to use LMWH as the standard-of-care treatment among VTE patients with cancer is based on clinical trials that compared LMWH to VKAs for the initial management of cancerassociated VTE. ${ }^{37-39}$ In the LITE trial, tinzaparin was associated with a lower rate of recurrent VTE and a similar rate of MB compared with VKA. ${ }^{35}$ In the $\mathrm{CATCH}$ trial, oncedaily tinzaparin was associated with a similar risk of recurrent VTE and MB, and a lower risk of CRNM bleeding compared with warfarin patients who bridged therapy with tinzaparin. ${ }^{36}$ In the CLOT trial, dalteparin had a significantly lower risk of recurrent VTE and a similar 


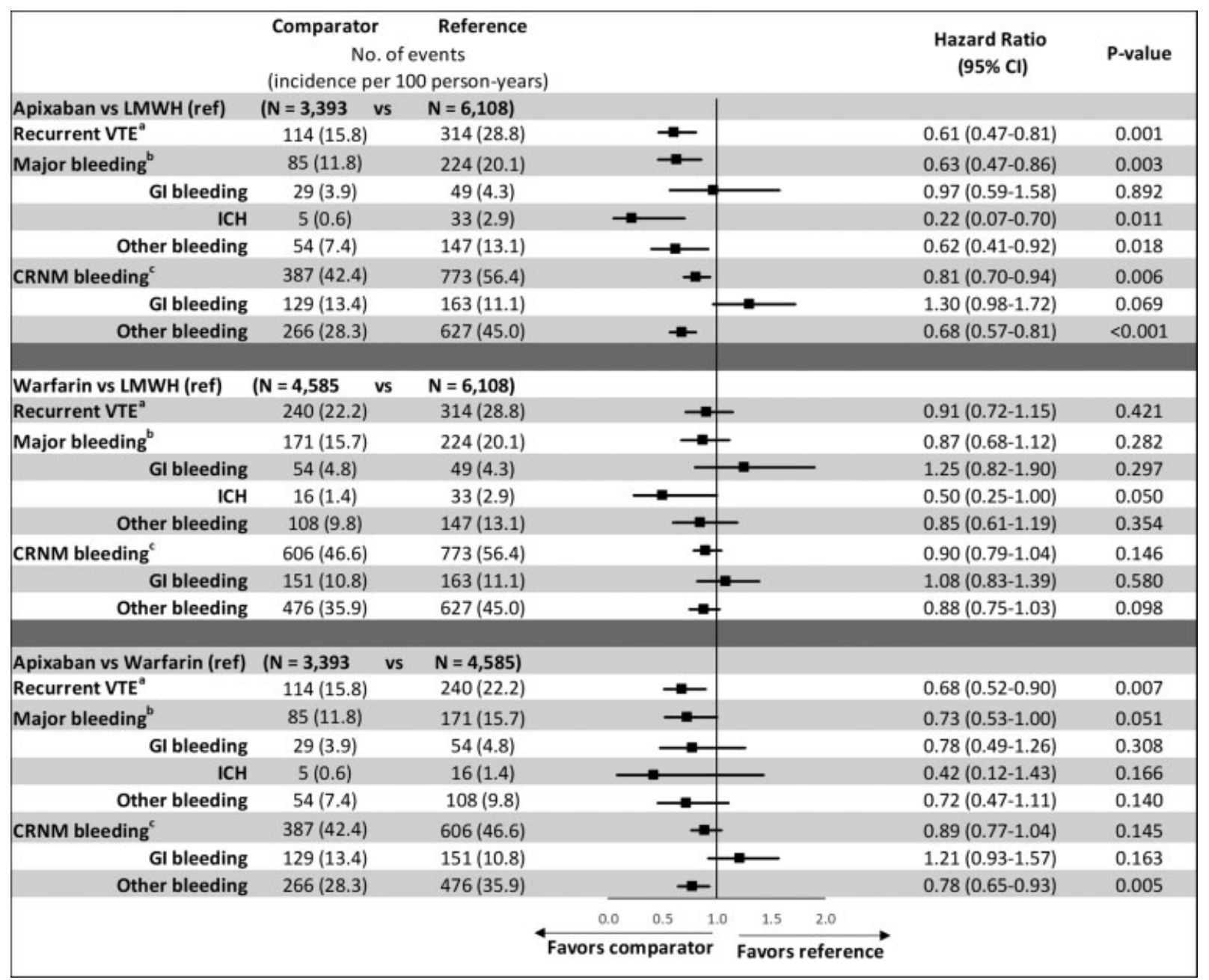

Fig. 4 Incidence rates and hazard ratios of recurrent VTE, major bleeding, and CRNM bleeding among VTE cancer patients prescribed apixaban, LMWH, or warfarin in the IPTW-weighted population censoring follow-up at 6 months. Cl, confidence interval; CRNM, clinically relevant nonmajor bleeding; $\mathrm{Gl}$, gastrointestinal; ICH, intracranial hemorrhage; LMWH, low-molecular-weight heparin; VTE, venous thromboembolism. ${ }^{a}$ Recurrent VTE was defined by first-listed diagnosis in the inpatient setting, excluding admissions that occurred within 7 days of the index VTE encounter. ${ }^{b}$ Major bleeding was defined by first-listed diagnosis in the inpatient setting and includes $\mathrm{Gl}$ bleeding, ICH, and major bleeding at other sites. 'CRNM bleeding includes $\mathrm{Gl}$ bleeding and CRNM bleeding at other sites. CRNM bleeding followed by major bleeding was excluded from the analysis.

risk of bleeding compared with oral anticoagulation (warfarin LMWH bridging therapy). ${ }^{37}$ While these clinical trials provide important information about the efficacy and safety of LMWH versus VKA in VTE cancer patients, the current study offers complementary evidence about the effects of PACs and OACs in routine clinical practice.

The last decade has seen an emergence of DOACs for the treatment of VTE. Clinical trials have demonstrated the effectiveness and safety of DOACs such as rivaroxaban and edoxaban in comparison to LWMH for VTE patients with cancer. ${ }^{18,40}$ A meta-analysis of randomized control trials comparing the efficacy and safety of DOACs (apixaban, dabigatran, edoxaban, rivaroxaban) with conventional therapy (heparin/VKA) in patients with VTE and mainly inactive cancer reported that recurrent VTE (3.9 vs. 6.0\%) and $\mathrm{MB}$ (3.2 vs. 4.2\%) were similar between the two groups. ${ }^{41}$ Another meta-analysis found that DOACs significantly reduced the risk of recurrent VTE by $35 \%$ compared with LMWH. ${ }^{42}$ However, DOACs were associated with a
$70 \%$ increase in the risk of MB compared with LMWH. ${ }^{40}$ There is limited evidence in the literature regarding the use of apixaban among VTE cancer patients. In the ADAM trial, which studied 300 cancer patients with VTE, apixaban was associated with very low rates of recurrent VTE and MB compared with dalteparin. ${ }^{22}$ The CARAVAGGIO study was the largest randomized controlled trial of a DOAC compared with LMWH; it found that apixaban was noninferior (recurrent VTE, HR: 0.63; 95\% CI: 0.37-1.07; $p<0.001$ for noninferiority) to dalteparin for the treatment of cancer-associated VTE without an increased risk of MB (HR: $0.82 ; 95 \% \mathrm{CI}: 0.40-1.69 ; p=0.60$ ), and, notably, there was no increase in major GI bleeding (HR: 1.05; 95\% CI: 0.44-2.50). ${ }^{21}$ Compared with the CARAVAGGIO study, this claims database analysis included a larger sample size and showed generally consistent trends for apixaban versus LMWH on recurrent VTE and MB. The current analysis provides complementary information to CARAVAGGIO. This combined evidence may help inform 


\begin{tabular}{|c|c|c|c|c|c|c|c|}
\hline \multicolumn{4}{|c|}{$\begin{array}{l}\text { Comparator Reference } \\
\text { No. of events } \\
\text { (incidence per } 100 \text { person-years) }\end{array}$} & & & \multirow[t]{2}{*}{$\begin{array}{c}\text { Hazard Ratio } \\
(95 \% \mathrm{Cl})\end{array}$} & \multirow[t]{2}{*}{ P-value } \\
\hline Apixaban vs LMWH (ref) & $(\mathrm{N}=3,393$ & vs & $N=6,108)$ & & & & \\
\hline Recurrent $\mathrm{VTE}^{\mathrm{a}}$ & $148(11.7)$ & & $396(23.2)$ & $\rightarrow-$ & & $0.59(0.47-0.75)$ & $<0.001$ \\
\hline Major bleeding ${ }^{b}$ & $123(9.7)$ & & $303(17.4)$ & $\rightarrow-$ & & $0.62(0.47-0.80)$ & $<0.001$ \\
\hline Gl bleeding & $39(3.0)$ & & $74(4.1)$ & $\rightarrow$ & - & $0.78(0.51-1.18)$ & 0.241 \\
\hline ICH & $5(0.4)$ & & $51(2.8)$ & $\rightarrow$ & & $0.15(0.05-0.43)$ & $<0.001$ \\
\hline Other bleeding & $80(6.2)$ & & $187(10.6)$ & $\rightarrow-$ & & $0.67(0.47-0.95)$ & 0.023 \\
\hline CRNM bleeding $^{c}$ & $427(36.8)$ & & $829(51.7)$ & $\rightarrow-$ & & $0.80(0.69-0.92)$ & 0.002 \\
\hline Gl bleeding & $141(11.3)$ & & $175(10.0)$ & & $\longrightarrow$ & $1.27(0.97-1.67)$ & 0.083 \\
\hline Other bleeding & $294(24.4)$ & & $672(41.1)$ & $\rightarrow$ & & $0.67(0.57-0.80)$ & $<0.001$ \\
\hline Warfarin vs LMWH (ref) & $(N=4,585$ & vs & $N=6,108)$ & & & & \\
\hline Recurrent $\mathrm{VTE}^{\mathrm{a}}$ & $326(16.0)$ & & $396(23.2)$ & $\rightarrow$ & - & $0.93(0.76-1.13)$ & 0.451 \\
\hline Major bleeding ${ }^{b}$ & $250(12.1)$ & & $303(17.4)$ & $\rightarrow-$ & + & $0.85(0.69-1.05)$ & 0.129 \\
\hline GI bleeding & $94(4.5)$ & & $74(4.1)$ & - & $\rightarrow$ & $1.27(0.91-1.79)$ & 0.165 \\
\hline $\mathrm{ICH}$ & $29(1.4)$ & & $51(2.8)$ & $\rightarrow-$ & & $0.54(0.31-0.94)$ & 0.028 \\
\hline Other bleeding & $138(6.6)$ & & $187(10.6)$ & $\rightarrow-$ & & $0.79(0.59-1.05)$ & 0.108 \\
\hline CRNM bleeding ${ }^{c}$ & $712(38.7)$ & & $829(51.7)$ & $\rightarrow$ & & $0.90(0.79-1.02)$ & 0.101 \\
\hline GI bleeding & $180(8.8)$ & & $175(10.0)$ & $\rightarrow$ & 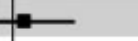 & $1.07(0.84-1.37)$ & 0.579 \\
\hline Other bleeding & $554(29.2)$ & & $672(41.1)$ & $\rightarrow$ & & $0.87(0.75-1.01)$ & 0.060 \\
\hline Apixaban vs Warfarin (ref) & $(\mathrm{N}=3,393$ & vs & $N=4,585)$ & & & & \\
\hline Recurrent $\mathrm{VTE}^{\mathrm{a}}$ & $148(11.7)$ & & $326(16.0)$ & $\rightarrow-$ & & $0.64(0.50-0.82)$ & $<0.001$ \\
\hline Major bleeding ${ }^{b}$ & $123(9.7)$ & & $250(12.1)$ & $\rightarrow-$ & & $0.72(0.55-0.95)$ & 0.018 \\
\hline GI bleeding & $39(3.0)$ & & $94(4.5)$ & $\rightarrow-$ & & $0.61(0.41-0.92)$ & 0.018 \\
\hline $\mathrm{ICH}$ & $5(0.4)$ & & $29(1.4)$ & $\rightarrow$ & & $0.27(0.09-0.81)$ & 0.019 \\
\hline Other bleeding & $80(6.2)$ & & $138(6.6)$ & $\longrightarrow$ & 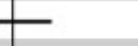 & $0.84(0.58-1.23)$ & 0.380 \\
\hline CRNM bleeding $^{c}$ & $427(36.8)$ & & $712(38.7)$ & $\Rightarrow$ & & $0.88(0.77-1.02)$ & 0.084 \\
\hline GI bleeding & $141(11.3)$ & & $180(8.8)$ & & $\Rightarrow$ & $1.17(0.92-1.50)$ & 0.209 \\
\hline Other bleeding & $294(24.4)$ & & $554(29.2)$ & $\rightarrow-$ & & $0.78(0.66-0.92)$ & 0.003 \\
\hline & & & & 0.0 & 1.5 & 2.0 & \\
\hline & & & & to & tor Favo & $\overrightarrow{\text { eference }}$ & \\
\hline
\end{tabular}

Fig. 5 Incidence rates and hazard ratios of recurrent VTE, major bleeding, and CRNM bleeding among VTE cancer patients prescribed apixaban, LMWH, or warfarin in the IPTW-weighted population during the entire follow-up. $\mathrm{Cl}$, confidence interval; CRNM, clinically relevant nonmajor bleeding; $\mathrm{Gl}$, gastrointestinal; ICH, intracranial hemorrhage; LMWH, low-molecular-weight heparin; VTE, venous thromboembolism. ${ }^{\mathrm{a}}$ Recurrent VTE was defined by first-listed diagnosis in the inpatient setting, excluding admissions that occurred within 7 days of the index VTE encounter. ${ }^{\mathrm{b}}$ Major bleeding was defined by first-listed diagnosis in the inpatient setting and includes $\mathrm{Gl}$ bleeding, ICH, and major bleeding at other sites. ${ }^{\mathrm{C} C R N M}$ bleeding includes $\mathrm{GI}$ bleeding and CRNM bleeding at other sites. CRNM bleeding followed by major bleeding was excluded from the analysis.

the shared decision-making process for the treatment of VTE among cancer patients.

Other retrospective observational studies comparing LMWH to OACs have also been conducted. A retrospective study using the electronic medical records of adult patients with cancer-related VTE reported similar risks of recurrent VTE and MB among patients who initiated DOACs versus LMWH. ${ }^{43}$ Another retrospective study conducted in a clinical setting that compared apixaban, enoxaparin, and rivaroxaban among patients with cancerassociated VTE reported that the risk of recurrent VTE and MB was similar across apixaban, enoxaparin, and rivaroxaban. ${ }^{44}$ Additionally, rivaroxaban was associated with an increased risk of CRNM bleeding compared with apixaban and enoxaparin. ${ }^{44}$

The use of LMWH remains challenging due to its risk-tobenefit ratio, cost, and inconvenience of use. This study found that apixaban had a significantly better effectiveness and safety profile compared with LMWH in patients with VTE and active cancer. Effectiveness and safety of anticoagulation treatment also depend on the risk stratification for VTE at the time of cancer diagnosis, which may play an important role in the assessment of the risk-to-benefit ratio among VTE cancer patients. ${ }^{45,46}$ Therefore, further studies are needed to evaluate the clinical outcomes of anticoagulation treatment among VTE cancer patients at high risk of recurrent VTE.

\section{Limitations}

As with all retrospective claims analyses, only associationsrather than causation-can be inferred from this study, and the results should be interpreted accordingly. The definition of recurrent VTE was based on inpatient claims with a 
primary (first-listed) ICD-9/10-CM diagnosis code for VTE (DVT or PE) with a positive predictive value ranging from 26 to $93 \%$, but this did not exclude patients with a VTE who were hospitalized for other reasons. Moreover, the presence of a diagnosis code on a medical claim may not indicate a positive presence of recurrent VTE or any disease, as the diagnosis code may be incorrectly coded or included as rule-out criteria rather than actual disease. Given the lack of clinical information in claims data, clinically adjudicated recurrent VTE diagnoses, cancer stage, laboratory test results (such as international normalized ratio values and serum creatinine/ creatinine clearance levels), and biomarkers (such as body weight) were not available. As the data were from U.S. commercial databases, results may not be generalizable to other populations. Duplicates were not excluded from the pooled database. However, prior literature reported only $0.5 \%$ duplicates between two databases. ${ }^{47}$ Hence, they should not impact the study results. Transfusion codes were one criterion used to identify $\mathrm{MB}$, which could have resulted in overestimation of MB since cancer patients are likely to receive transfusions for other reasons. However, the codes have been derived from a validated MB definition which had a positive predictive value of $\geq 89 \%{ }^{48}$ Since hemoglobin values were not known and significant hemoglobin drop cannot be identified from the databases, MB could have been underestimated. The algorithm and ICD codes used to identify CRNM bleeding have not been validated in the literature. However, the definition used in this claims data analysis attempted to follow the definition suggested by theISTH. ${ }^{49}$ Nonetheless, proportions of patients with CRNM bleeding may be under- or overreported in the present study due to misclassification. Among patients who bridged therapy, those who had a recurrent VTE or MB event between the time of LMWH and warfarin initiation were excluded. However, only 52 patients were excluded using the above criteria and hence this exclusion should not impact the overall study results. The commercial databases do not have complete death information for the patients; hence, we could not evaluate mortality and fatal recurrent VTE among this population, and mortality may be a competing risk in this population. Medications prescribed during hospitalization could not be identified in the commercial databases. Finally, the results may not be generalizable to the entire U.S. VTE cancer population, since uninsured patients or patients with governmental insurances such as Medicare, Medicaid, and Veterans Affairs were not evaluated.

\section{Conclusion}

This is the largest retrospective claims database study comparing apixaban, LMWH, and warfarin among patients with VTE and active cancer. Apixaban was found to be associated with lower risks of recurrent VTE, MB, and CRNM bleeding compared with LMWH. Apixaban was also associated with a lower risk of recurrent VTE compared with warfarin. Warfarin was associated with similar risks of recurrent VTE, MB, and CRNM bleeding compared with LMWH patients. Together with randomized controlled trial data, this study may be helpful for clinicians in evaluating different anticoagulation treatments for patients with VTE and active cancer.

\section{What is known about this topic?}

- Venous thromboembolism (VTE) is a leading cause of death in cancer patients receiving cancer therapy.

- Treating VTE patients with cancer is challenging due to an increased risk of recurrences and bleeding associated with anticoagulant use as well as potential drugdrug interactions.

- There is a lack of real-world evidence comparing the effectiveness and safety of low-molecular-weight heparin (LMWH) with warfarin or direct-acting oral anticoagulants (DOACs) such as apixaban among patients with VTE and active cancer.

\section{What does this paper add?}

- This study evaluates the risk of recurrent VTE, major bleeding (MB), and clinically relevant nonmajor (CRNM) bleeding among patients with VTE and active cancer prescribed apixaban, LMWH, or warfarin.

- Patients with VTE and active cancer who initiated apixaban had a significantly lower risk of recurrent VTE, MB, and CRNM bleeding compared with LMWH patients.

- Apixaban patients also had a lower risk of recurrent VTE compared with warfarin patients.

\section{Funding}

This research was funded by Pfizer Inc. and Bristol-Myers Squibb Company.

Conflict of Interest

A.C. received research support from Pfizer Inc. and BristolMyers Squibb Company. A.K. and J.S. are employed by SIMR, Inc., a paid consultant to Pfizer Inc. and BristolMyers Squibb Company in connection with the development of the manuscript. T.L., P.H., J.M., D.W., and X.L. are employees of Pfizer Inc., a study sponsor. G.W. and L.R. are employees of Bristol-Myers Squibb Company, a study sponsor.

\section{References}

1 Khorana AA. Venous thromboembolism and prognosis in cancer. Thromb Res 2010;125(06):490-493

2 Heit JA, O'Fallon WM, Petterson TM, et al. Relative impact of risk factors for deep vein thrombosis and pulmonary embolism: a population-based study. Arch Intern Med 2002;162(11): $1245-1248$

3 Gussoni G, Frasson S, La Regina M, Di Micco P, Monreal MRIETE Investigators. Three-month mortality rate and clinical predictors in patients with venous thromboembolism and cancer. 
Findings from the RIETE registry. Thromb Res 2013;131(01): 24-30

4 Khorana AA, Francis CW, Culakova E, Fisher RI, Kuderer NM, Lyman GH. Thromboembolism in hospitalized neutropenic cancer patients. J Clin Oncol 2006;24(03):484-490

5 Chew HK, Wun T, Harvey D, Zhou H, White RH. Incidence of venous thromboembolism and its effect on survival among patients with common cancers. Arch Intern Med 2006;166 (04):458-464

6 Holm T, Singnomklao T, Rutqvist LE, Cedermark B. Adjuvant preoperative radiotherapy in patients with rectal carcinoma. Adverse effects during long term follow-up of two randomized trials. Cancer 1996;78(05):968-976

7 Trinh VQ Karakiewicz PI, Sammon J, et al. Venous thromboembolism after major cancer surgery: temporal trends and patterns of care. JAMA Surg 2014;149(01):43-49

8 Cohen AT, Katholing A, Rietbrock S, Bamber L, Martinez C. Epidemiology of first and recurrent venous thromboembolism in patients with active cancer. A population-based cohort study. Thromb Haemost 2017;117(01):57-65

9 Elting LS, Escalante CP, Cooksley C, et al. Outcomes and cost of deep venous thrombosis among patients with cancer. Arch Intern Med 2004;164(15):1653-1661

10 Prandoni P, Lensing AW, Piccioli A, et al. Recurrent venous thromboembolism and bleeding complications during anticoagulant treatment in patients with cancer and venous thrombosis. Blood 2002;100(10):3484-3488

11 Kearon C, Akl EA, Ornelas J, et al. Antithrombotic therapy for VTE disease: CHEST guideline and expert panel report. Chest 2016; 149(02):315-352

12 Konstantinides SV, Meyer G, Becattini C, et al; The Task Force for the diagnosis and management of acute pulmonary embolism of the European Society of Cardiology (ESC). 2019 ESC Guidelines for the diagnosis and management of acute pulmonary embolism developed in collaboration with the European Respiratory Society (ERS): the task force for the diagnosis and management of acute pulmonary embolism of the European Society of Cardiology (ESC). Eur Respir J 2019;54(03):1-61

13 Farge D, Bounameaux H, Brenner B, et al. International clinical practice guidelines including guidance for direct oral anticoagulants in the treatment and prophylaxis of venous thromboembolism in patients with cancer. Lancet Oncol 2016;17(10): e452-e466

14 Streiff MB, Holmstrom B, Angelini D, et al. NCCN guidelines insights: cancer-associated venous thromboembolic disease, version 2.2018. J Natl Compr Canc Netw 2018;16(11):1289-1303

15 Lee AYY, Peterson EA. Treatment of cancer-associated thrombosis. Blood 2013;122(14):2310-2317

16 Key NS, Khorana AA, Kuderer NM, et al. Venous thromboembolism prophylaxis and treatment in patients with cancer: ASCO clinical practice guideline update. J Clin Oncol 2020;38(05):496-520

17 Khorana AA, Yannicelli D, McCrae KR, et al. Evaluation of US prescription patterns: are treatment guidelines for cancer-associated venous thromboembolism being followed? Thromb Res 2016;145:51-53

18 Raskob GE, van Es N, Verhamme P, et al; Hokusai VTE Cancer Investigators. Edoxaban for the treatment of cancer-associated venous thromboembolism. N Engl J Med 2018;378(07):615-624

19 Win KZ, Wilson N, Stenehjem DD, Tanner N, Rodgers GM, Gilreath $\mathrm{J}$. Effectiveness and safety of rivaroxaban in treatment of venous thromboembolism in cancer patients. Blood 2015;126:2319

20 Khorana AA, Noble S, Lee AYY, et al. Role of direct oral anticoagulants in the treatment of cancer-associated venous thromboembolism: guidance from the SSC of the ISTH. J Thromb Haemost 2018;16(09):1891-1894

21 Agnelli G, Becattini C, Meyer G, et al; Caravaggio Investigators. Apixaban for the treatment of venous thromboembolism associated with cancer. N Engl J Med 2020;382(17):1599-1607
22 McBane RD II, Wysokinski WE, Le-Rademacher JG, et al. Apixaban and dalteparin in active malignancy-associated venous thromboembolism: the ADAM VTE trial. J Thromb Haemost 2020;18(02): 411-421

23 Pan X, Kachroo S, Liu X, et al. Real world discontinuation among early users of apixaban, dabigatran, rivaroxaban or warfarin among atrial fibrillation patients newly initiated on anticoagulation therapy: Tell of first 200 days [abstract]. Eur Heart J [serial online] 2014;35(Abstract Supplement):897

24 Teutsch C, Huisman MV, Lip GY, et al. Persistence with dabigatran therapy for stroke prevention in patients with non-valvular atrial fibrillation: the Gloria-AF Registry. Blood 2016;128:2616

25 Tamariz L, Harkins T, Nair V. Mini-sentinel systematic evaluation of health outcome of interest definitions for studies using administrative data venous thromboembolism report. Mini-Sentinel 2011. Available at: https://www.sentinelinitiative.org/sites/default/files/surveillance -tools/validations-literature/Mini-Sentinel-HOI-Evidence-ReviewVenous-Thromboembolism-Report.pdf. Accessed August 20, 2020

26 Fang MC, Fan D, Sung SH, et al. Validity of using inpatient and outpatient administrative codes to identify acute venous thromboembolism: the CVRN VTE study. Med Care 2017;55(12):e137-e143

27 Khorana AA, McCrae KR. Risk stratification strategies for cancerassociated thrombosis: an update. Thromb Res 2014;133 (Suppl 2):S35-S38

28 Austin PC. Variance estimation when using inverse probability of treatment weighting (IPTW) with survival analysis. Stat Med 2016;35(30):5642-5655

29 Comorbidity Index Overview NCl. Healthcaredelivery.cancer.gov [online]. Available at: https://healthcaredelivery.cancer.gov/seermedicare/considerations/comorbidity.html. Accessed March 19, 2020

30 Austin PC, Stuart EA. Moving towards best practice when using inverse probability of treatment weighting (IPTW) using the propensity score to estimate causal treatment effects in observational studies. Stat Med 2015;34(28):3661-3679

31 Thoemmes F, Ong AD. A primer on inverse probability of treatment weighting and marginal structural models. Emerg Adulthood 2016;4(01):40-59

32 Xu S, Ross C, Raebel MA, Shetterly S, Blanchette C, Smith D. Use of stabilized inverse propensity scores as weights to directly estimate relative risk and its confidence intervals. Value Health 2010; 13(02):273-277

33 Mahé I, Chidiac J, Helfer H, Noble S. Factors influencing adherence to clinical guidelines in the management of cancer-associated thrombosis. J Thromb Haemost 2016;14(11):2107-2113

34 Wittkowsky AK. Barriers to the long-term use of low-molecular weight heparins for treatment of cancer-associated thrombosis. J Thromb Haemost 2006;4(09):2090-2091

35 Bauer KA. Pros and cons of new oral anticoagulants. Hematology (Am Soc Hematol Educ Program) 2013;2013:464-470

36 Mekaj YH, Mekaj AY, Duci SB, Miftari EI. New oral anticoagulants: their advantages and disadvantages compared with vitamin $\mathrm{K}$ antagonists in the prevention and treatment of patients with thromboembolic events. Ther Clin Risk Manag 2015;11:967-977

37 Hull RD, Pineo GF, Brant RF, et al; LITE Trial Investigators. Longterm low-molecular-weight heparin versus usual care in proximal-vein thrombosis patients with cancer. Am J Med 2006;119 (12):1062-1072

38 Lee AYY, Kamphuisen PW, Meyer G, et al; CATCH Investigators. Tinzaparin vs warfarin for treatment of acute venous thromboembolism in patients with active cancer: a randomized clinical trial. JAMA 2015;314(07):677-686

39 Lee AY, Levine MN, Baker RI, et al; Randomized Comparison of Low-Molecular-Weight Heparin versus Oral Anticoagulant Therapy for the Prevention of Recurrent Venous Thromboembolism in Patients with Cancer (CLOT) Investigators. Low-molecularweight heparin versus a coumarin for the prevention of recurrent venous thromboembolism in patients with cancer. $\mathrm{N}$ Engl J Med 2003;349(02):146-153 
40 Young AM, Marshall A, Thirlwall J, et al. Comparison of an oral factor Xa inhibitor with low molecular weight heparin in patients with cancer with venous thromboembolism: Results of a randomized trial (SELECT-D). J Clin Oncol 2018;36(20):2017-2023

41 Vedovati MC, Germini F, Agnelli G, Becattini C. Direct oral anticoagulants in patients with VTE and cancer: a systematic review and meta-analysis. Chest 2015;147(02):475-483

42 Li A, Garcia DA, Lyman GH, Carrier M. Direct oral anticoagulant (DOAC) versus low-molecular-weight heparin (LMWH) for treatment of cancer associated thrombosis (CAT): A systematic review and meta-analysis. Thromb Res 2019;173:158-163

43 Ross JA, Miller MM, Rojas Hernandez CM. Comparative effectiveness and safety of direct oral anticoagulants (DOACs) versus conventional anticoagulation for the treatment of cancer-related venous thromboembolism: a retrospective analysis. Thromb Res 2017;150:86-89

44 Wysokinski WE, Houghton DE, Casanegra AI, et al. Comparison of apixaban to rivaroxaban and enoxaparin in acute cancer-associated venous thromboembolism. Am J Hematol 2019;94(11):1185-1192
45 Khorana AA, Connolly GC. Assessing risk of venous thromboembolism in the patient with cancer. J Clin Oncol 2009;27(29): 4839-4847

46 Ay C, Dunkler D, Marosi C, et al. Prediction of venous thromboembolism in cancer patients. Blood 2010;116(24):5377-5382

47 Broder MS, Neary MP, Chang E, Cherepanov D, Katznelson L. Treatments, complications, and healthcare utilization associated with acromegaly: a study in two large United States databases. Pituitary 2014;17(04):333-341

48 Cunningham A, Stein CM, Chung CP, Daugherty JR, Smalley WE, Ray WA. An automated database case definition for serious bleeding related to oral anticoagulant use. Pharmacoepidemiol Drug Saf 2011;20(06):560-566

49 Kaatz S, Ahmad D, Spyropoulos AC, Schulman SSubcommittee on Control of Anticoagulation. Definition of clinically relevant nonmajor bleeding in studies of anticoagulants in atrial fibrillation and venous thromboembolic disease in non-surgical patients: communication from the SSC of the ISTH. J Thromb Haemost 2015;13(11):2119-2126 